\title{
Hypofractionated stereotactic radiation therapy for recurrent glioblastoma: single institutional experience
}

\author{
Patrizia Ciammella ${ }^{1 *}$, Ala Podgornii ${ }^{1}$, Maria Galeandro ${ }^{1}$, Nunziata D'Abbiero ${ }^{1}$, Anna Pisanello², Andrea Botti ${ }^{3}$, \\ Elisabetta Cagni ${ }^{3}$, Mauro lori $^{3}$ and Cinzia lotti ${ }^{1}$
}

\begin{abstract}
Background: Glioblastoma (GBM) is the most common malignant primary brain tumor in adults. Tumor control and survival have improved with the use of radiotherapy (RT) plus concomitant and adjuvant chemotherapy, but the prognosis remain poor. In most cases the recurrence occurs within 7-9 months after primary treatment. Currently, many approaches are available for the salvage treatment of patients with recurrent GBM, including resection, re-irradiation or systemic agents, but no standard of care exists.

Methods: We analysed a cohort of patients with recurrent GBM treated with frame-less hypofractionated stereotactic radiation therapy with a total dose of $25 \mathrm{~Gy}$ in 5 fractions.

Results: Of 91 consecutive patients with newly diagnosed GBM treated between 2007 and 2012 with conventional adjuvant chemo-radiation therapy, 15 underwent salvage RT at recurrence. The median time interval between primary RT and salvage RT was 10.8 months (range, 6-54 months). Overall, patients undergoing salvage RT showed a longer survival, with a median survival of 33 vs. 9.9 months $(p=0.00149)$. Median overall survival (OS) from salvage RT was 9.5 months. No patients demonstrated clinically significant acute morbidity, and all patients were able to complete the prescribed radiation therapy without interruption.

Conclusion: Our results suggest that hypofractionated stereotactic radiation therapy is effective and safe in recurrent GBM. However, until prospective randomized trials will confirm these results, the decision for salvage treatment should remain individual and based on a multidisciplinary evaluation of each patient.
\end{abstract}

Keywords: Recurrent glioblastoma, Stereotactic radiation therapy, Re-irradiation, Acute toxicity

\section{Introduction}

The current standard of care for glioblastoma (GBM) is concurrent Temozolomide (TMZ) and conventional radiotherapy followed by 6 maintenance cycles of adjuvant TMZ. Despite standard of care therapy, recurrence rates remain high $(>90 \%)$ with a median overall survival (OS) of 15-18 months [1].

In recent years a lot of mono-therapy or combination chemotherapeutic strategies have been evaluated in patients with recurrent or progressive GBM. The alkylant

\footnotetext{
* Correspondence: patrizia.ciammella@asmn.re.it

'Radiation Therapy Unit, Department of Oncology and Advanced Technology, Azienda Ospedaliera ASMN, Istituto di Ricovero e Cura a Carattere Scientifico, Viale Risorgimento 80, 42123 Reggio Emilia, Italy Full list of author information is available at the end of the article
}

agents (nitrosoureas, procarbazine), used in the past as first-line treatment, are used today as second-line therapy for recurrence or progression. Overall, the 6-months progression-free survival (PFS) and median OS with second-line therapy with alkylant agents ranged from 13 to $24 \%$ and from 5 to 11 months, respectively [2-8]. Numerous trials have evaluated the efficacy and safety of TMZ as a monotherapy for recurrent GBM in patients previously treated with chemotherapy, mostly nitrosureabased [9-15]. Some recent studies evaluated in TMZpretreated patients a second-line TMZ based therapy [16-21]. In one of these studies, the TMZ resulted more efficacious than procarbazine (PFS at 6 months $=21 \%$ vs $8 \%$ ) [16]. Six recent trials of TMZ-pretreated patients evaluated TMZ re-challenge [17-21]. Unfortunately, considering the

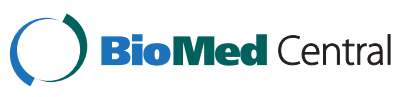


small numbers of patients included in these studies and the wide range of TMZ regimen tested, there was no evidence that one regimen was advantageous over another, and despite some improvements in PFS none of them was associated with a better survival. The repeated surgery is feasible only in few cases of recurrent GBM and is not recommended for patients with involvement of prespecified eloquent/critical brain regions [22]. The data related to reoperation have been derived from retrospective studies, but it is known that positive prognostic factors of reoperation for recurrent GBM are a younger age $(\leq 70$ years $)$, a smaller tumor volume $(\leq 50 \mathrm{~cm} 3)$ and preoperative KPS greater than $80 \%[22,23]$.

Today, for recurrent GBM the re-irradiation remains a palliative option for a selected group of patients, i.e. those with a KPS greater than $60 \%$, a tumor size of up to 40 $\mathrm{mm}$, and a time to progression from the surgery of at least 6 months [24]. Usually, a stereotactic radiotherapy technique is applied [25], but the fractionation schedule and the prescribed total dose are various and no randomized trials are available. Both single-fraction stereotactic radiosurgery (SRS) and fractionated stereotactic radiotherapy (FSRT) have been reported to have minimal toxicity, with median survivals of 6-10 months after SRS [26-30] or FSRT [31-33]. Recently, some studies also have evaluated the safety and efficacy of the combination of fractionated RT and concurrent chemotherapy in recurrent high-grade gliomas showing a median OS and PFS from reirradiation of 8-13 and 5-8 months, respectively [34-37].

Our retrospective analysis focused on the efficacy and toxicity of a hypofractionated stereotactic radiotherapy for recurrent GBM in a cohort of patients previously treated with standard therapy.

\section{Methods and materials}

This is a retrospective study of efficacy and safety of hypofractionated stereotactic radiation therapy (SRT) delivered at 5 fractions of 5 Gy each for management of recurrent intracranial GBM. All patients previously underwent a conventional adjuvant chemo-radiation therapy according to Stupp protocol after a maximal surgical resection for a newly diagnosed GBM. The histopathological diagnosis of primary GBM was established according to the current World Health Organization Criteria [38].

The protocol was approved by the local Ethics committee and informed consent was obtained for all patients.

\section{Recurrence definition}

The diagnosis of tumor recurrence was based on the joint opinion of the neuro-radiologist, neurosurgeon, radiation oncologist and neuro-oncologist, and was defined as appearance of new contrast-enhanced lesion(s) on T1-weichted MRI or an increase of $25 \%$ or more of the volume of the initial enhanced lesion(s). In case of doubt between tumor recurrence and pseudo-progression a MR imaging was repeated after one month. Recurrences were defined, as suggested by Lee et at [39], as " in-field" if $>80 \%$ of the tumour recurrence resided within the prescription $95 \%$ isodose surface, and "marginal" if $20 \%$ to $80 \%$ of the lesion was inside the $95 \%$ isodose surface. In all other cases, recurrences were defined as outside of the radiation field. At the time of recurrence, all patients were evaluated for salvage treatment, which included reresection of the tumor, hypo-fractionated radiation therapy, chemotherapy, or combined approaches.

\section{Re-irradiation protocol}

The indication for re-irradiation at tumor recurrence was evaluated by the interdisciplinary neuro-oncology team and based on: the patient clinical condition (i.e. KPS> 60), the lesion location and the spread of disease (patients with multifocal spread of disease were excluded). All patients undergoing re-irradiation were immobilized in customized thermoplastic shells for CT and MR simulation. The GTV was delineated on the basis of the contrast-enhancing tumor on T1-weighted MRI registered with CT images. For the image registration an automatic fusion algorithm was used; anyway the resulted registration was subsequently checked by mean of anatomic markers and, if needed, manually adjusted. The PTV was generated by applying to GTV an isotropic expansion of 3-5 $\mathrm{mm}$. The critical structures and previously high dose irradiated volumes were contoured. All patients were treated with hypo-fractionated stereotactic radiation therapy with multiple no-coplanar beams using on a standard 6-MV linear accelerator (LINAC). A total dose of 25 Gy prescribed to the $70 \%$ isodose line and delivered in 5 consecutive fractions was delivered. A daily pre-treatment verification with orthogonal fields was performed.

\section{Follow-up protocol}

All patients were evaluated at four weeks, 12 weeks and once every 3 months after the salvage treatment. The work-up included history and physical examination (with particular regard to KPS, neurological status and toxicity assessment), blood tests and contrast-enhanced brain MRI. No specific quality-of-life questionnaire was administered. Haematologic and no-haematological toxicities were graded according to Common Terminology Criteria for Adverse Events. Other investigations were not performed unless were clinically indicated.

\section{Statistical analysis}

Statistical analysis were performed using the R statistical software (http://www.R-project.org), particularly the survival package. Patient characteristics were compared with Fisher-test and a twosided significance level was 
chosen at 0.05. Survival curves were estimated using the Kaplan-Meier method. The p-values estimated are those for a two tailed test and the significance level was chosen to be $5 \%$. Cox regression analysis was used for univariate analysis and parameters were tested for proportionally and categorical covariates compared by log-rank test. A multivariate Cox proportional hazards analysis was used to evaluate prognostic factors and treatment with respect to the overall survival from initial diagnosis and from time of recurrence. Variables included in the model were selected when statistically significant in univariate analysis. Data are presented as 1-mont actuarial hazard ratios (HR) with 95\% confidence intervals, unless otherwise mentioned.

\section{Results}

Between 2007 and 2012 we treated with standard chemoradiation therapy according to Stupp protocol 91 patients with newly diagnosed GBM. With mean follow-up of 13.9 months (range 1-63 months), the median OS was 15 months, with a PFS of 9 months. In this populations of patients, the age ( $\leq 65$ years), extent of resection, KPS, RPA class and O6-methylguanine-DNA-methyltransferase (MGMT) methylation were significantly associated with OS.

The pattern of recurrences was analysed in 83 patients who had recurrences. Recurrence occurred "in-field" in 61 patients (73\%), at RT field margin in 7 patients (9\%) and "out-field" in 15 patients (18\%). No patients had CSF/ spinal or distant disease recurrence. No correlation between extent of surgery and site of relapse was seen $(>0.05)$. Progression-free survival did not differ significantly between patients with regional or marginal progression and patients with distant recurrences. Comparison of the investigated clinical factors did not differ significantly between the two groups ("in-field" vs "out-field"). No correlation between tumor location, tumor side, extent of primary surgery and pattern of recurrence was found.

At the time of recurrence, re-surgery was performed in 6 patients and second line chemotherapy in 37 patients. The salvage re-irradiation was performed in 9 of 58 patients with regional or marginal tumor progression and in 6 of 13 patients with distant recurrence. The remaining patients with tumor recurrence not eligible for salvage therapies (due to old age, poor performance status, severe comorbidities, patient choice) have received best supportive care. Table 1 shows the comparison between the different types of approaches for recurrent GBM (re-irradiation, re-surgery, second-line chemotherapy and best supportive care) in terms of patients' characteristics and clinical outcomes. The median time interval between primary $\mathrm{RT}$ and re-irradiation was 10.8 months (range, 6-54 months). No patients demonstrated clinically significant acute morbidity, and all patients were able to complete the prescribed treatment without interruption. No patient required hospitalization or surgery for early acute or delayed toxicity. Neurological deterioration occurred in two patients at 1 and 3 months after re-irradiation and was managed successfully with dexamethasone. Log-rank test revealed that the patients undergoing re-irradiation showed a longer survival compared to those treated with best supportive care, with a median overall survival from primary GBM diagnosis of 33 vs 9.9 months $(\mathrm{p}=0.0015)$. As well the patients treated with re-surgery or second line chemotherapy showed a longer survival than untreated patients, with a median OS from diagnosis of 17 months. The median time of recurrence from primary GBM diagnosis was statistically significant higher in the re-irradiation group when compared to all patients (19 months, 10 months, 8 months and 5 months in RT, surgery, chemotherapy and best supportive care, respectively, $\mathrm{p}=0.00003)$. This finding may explain the higher overall survival of patients undergoing re-irradiation. The median survival from recurrence was 9.5 months, 5.5 months and 2.5 months for RT, chemotherapy and best supportive care group, respectively. This difference was statistically significant between RT and best supportive care group $(\mathrm{p}=0.000001)$, between chemotherapy and best supportive care group $(\mathrm{p}=0.00007)$ and between RT and second-line chemotherapy group $(p=0.049)$. The Figures 1 and 2 show the Kaplan-Meier curves for OS from primary GBM diagnosis and from recurrence, comparing those treated with RT, surgery, chemotherapy and best supportive care.

The clinical characteristics of the patients in the four treatment groups were similar, except for the median age that was significantly lower in the RT group $(\mathrm{p}<0.05)$. The patients who underwent re-irradiation and re-surgery showed better clinical conditions (KPS) compared with patients treated with second-line chemotherapy or untreated (best supportive care), but the difference did not reach statistical significance.

In all re-treated patients, the multivariate Cox proportional hazard analysis confirmed the negative prognostic effect of the older age [hazard ratio (HR): 4.1 (95\%CI: $1.5-10.3), \mathrm{p}=0.005]$ and the biopsy alone [HR: 4.5 (95\%CI: $1.4-14.4), \mathrm{p}=0.011]$ on the OS. No correlation was found between the other analysed factors (sex, MGMT methylation, RPA class, primary tumor side, primary tumor volume, KPS at diagnosis and KPS at recurrence) and OS. In all recurrent patients there was no correlation between OS from recurrence and type of recurrence ("in-field" or "out-field"), though in the group of re-irradiated patients with "in-field" progression had a mild tendency to better OS comparing with patients with "out-field". Surprisingly, in the reirradiated group, the age alone (with cut-off of 60 years) showed a correlation with OS [HR: $11.92(95 \%$ CI: 1.1-135), $\mathrm{p}=0.0045]$. None of the others assessed factors reached statistical significance. 
Table 1 Comparison of clinical factors and outcome variables in patients with recurrent glioblastoma treated with different approaches

\begin{tabular}{|c|c|c|c|c|c|}
\hline \multirow[b]{2}{*}{ Characteristics } & \multicolumn{5}{|c|}{ Salvage treatment } \\
\hline & $\begin{array}{l}\text { Re-irradiation } \\
\quad(\mathrm{N}=15)\end{array}$ & $\begin{array}{l}\text { Re-surgery } \\
\qquad(\mathrm{N}=6)\end{array}$ & $\begin{array}{l}\text { Chemotherapy } \\
\qquad(\mathrm{N}=37)\end{array}$ & $\begin{array}{l}\text { Best supportive care } \\
\qquad(\mathrm{N}=25)\end{array}$ & $p$ Value \\
\hline \multicolumn{6}{|l|}{ Age (years) } \\
\hline Median & 51.5 & 65 & 66 & 66 & RT vs no-RT 0.002 \\
\hline Range & $41-73$ & $55-73$ & $49-72$ & $55-79$ & \\
\hline$<60$ years & 5 & 3 & 21 & 20 & \\
\hline$<=60$ years & 10 & 3 & 16 & 5 & 0.02 \\
\hline \multicolumn{6}{|l|}{ Gender, N0. (\%) } \\
\hline Men & $11(73 \%)$ & $4(67 \%)$ & $22(59 \%)$ & $9(38 \%)$ & 0.33 \\
\hline Women & $4(27 \%)$ & $2(33 \%)$ & $15(41 \%)$ & $16(62 \%)$ & \\
\hline \multicolumn{6}{|l|}{ Primary Surgery, NO. (\%) } \\
\hline Total & $11(73 \%)$ & $4(67 \%)$ & $18(49 \%)$ & $10(39 \%)$ & 0.18 \\
\hline Subtotal & $3(20 \%)$ & $2(33 \%)$ & $11(30 \%)$ & $4(15 \%)$ & \\
\hline Biopsy & $1(7 \%)$ & 0 & $8(21 \%)$ & $11(46 \%)$ & \\
\hline \multicolumn{6}{|l|}{ Karnofsky performance status at diagnosis (\%) } \\
\hline Median & 90 & 90 & 90 & 90 & n.s \\
\hline Range & $80-100$ & $80-100$ & $70-100$ & $70-100$ & \\
\hline \multicolumn{6}{|l|}{ RPA classification at diagnosis, NO. (\%) } \\
\hline IV & $9(60 \%)$ & $5(83 \%)$ & $18(50 \%)$ & $13(54 \%)$ & \\
\hline V & $5(33 \%)$ & $1(17 \%)$ & $8(20 \%)$ & $6(23 \%)$ & \\
\hline $\mathrm{Vl}$ & $1(7 \%)$ & 0 & $11(30 \%)$ & $6(23 \%)$ & \\
\hline \multicolumn{6}{|l|}{ MGMT Methylation, NO. (\%) } \\
\hline Yes & $7(47 \%)$ & $4(67 \%)$ & $12(33 \%)$ & $10(38 \%)$ & 0.47 \\
\hline No & $5(33 \%)$ & $2(33 \%)$ & $20(54 \%)$ & $13(54 \%)$ & \\
\hline Unknown & $3(20 \%)$ & 0 & $5(13 \%)$ & $2(8 \%)$ & \\
\hline \multicolumn{6}{|l|}{ Primary Tumor location, NO (\%) } \\
\hline Frontal & $3(20 \%)$ & $3(50 \%)$ & $12(34 \%)$ & $10(39 \%)$ & 0.72 \\
\hline Temporal & $4(27 \%)$ & $3(50 \%)$ & $13(35 \%)$ & $6(23 \%)$ & \\
\hline Parietal & $6(40 \%)$ & 0 & $8(21 \%)$ & $6(23 \%)$ & \\
\hline Occipital & $2(13 \%)$ & 0 & $4(10 \%)$ & $3(15 \%)$ & \\
\hline \multicolumn{6}{|l|}{ Primary Tumor side, NO (\%) } \\
\hline Left & $8(53 \%)$ & $4(67 \%)$ & $20(54 \%)$ & $14(54 \%)$ & 0.96 \\
\hline Right & $7(47 \%)$ & $2(33 \%)$ & $17(46 \%)$ & $11(46 \%)$ & \\
\hline \multicolumn{6}{|l|}{ Type of recurrence, NO (\%) } \\
\hline In-field & $9(60 \%)$ & $5(83 \%)$ & $32(86 \%)$ & $19(77 \%)$ & \\
\hline Out-field & $6(40 \%)$ & $1(17 \%)$ & $5(14 \%)$ & $6(23 \%)$ & 0.19 \\
\hline \multicolumn{6}{|l|}{ Karnofsky performance status at recurrence (\%) } \\
\hline Median & 90 & 90 & 80 & 80 & n.s. \\
\hline Range & $80-100$ & $80-100$ & $70-100$ & $70-100$ & \\
\hline Median OS from primary diagnosis (months) & 33 & 17 & 17 & 9.9 & RT vs no-RT 0.001 \\
\hline \multirow[t]{2}{*}{ Cox regression analysis $O R$ coef $f-p$} & 0.102 & 0.268 & 0.331 & 1 & \\
\hline & $1.4 \mathrm{e}-07$ & 0.016 & 0.00018 & - & \\
\hline
\end{tabular}


Table 1 Comparison of clinical factors and outcome variables in patients with recurrent glioblastoma treated with different approaches (Continued)

\begin{tabular}{|c|c|c|c|c|c|}
\hline \multicolumn{6}{|c|}{ Time between primary therapy and salvage treatment } \\
\hline Median (months) & 10.8 & 10 & 8 & 5 & 0.003 \\
\hline Range (months) & $6-54$ & $7-24$ & $7-21$ & $2-11$ & \\
\hline Median OS from recurrence(months) & 9.5 & 5.5 & 5.5 & 2.5 & RT vs no-RT 0.001 \\
\hline \multirow[t]{2}{*}{ Cox regression analysis $O R$ coef $f-p$} & 0.16 & 0.34 & 0.33 & 1 & \\
\hline & 0.000016 & 0.052 & 0.0015 & - & \\
\hline
\end{tabular}

\section{Discussion}

This retrospective study reports the results of a series of patients affected by GBM treated with conventional chemo-radiation therapy ad diagnosis and who underwent various salvage treatment for their tumor recurrence. The principal focus of our analysis is to evaluate the feasibility and safety of re-irradiation at tumor progression in patients previously irradiated. It is well known that the tumor control and survival in patients with GBM have been improved by the use of radiotherapy plus concomitant and adjuvant TMZ. Unfortunately, the prognosis remain poor with very few long-term survivors. In most cases the recurrence occurs within 7-9 months from initial diagnosis [40-42]. Up to $90 \%$ of all GBMs relapse in close proximity to the resection cavity or to the target volume of postoperative radiotherapy [43,44]. Currently, many approaches are available for the salvage treatment of patients with recurrent GBM after initial chemo-radiation therapy, including resection, reirradiation or systemic agents, but no standard of care

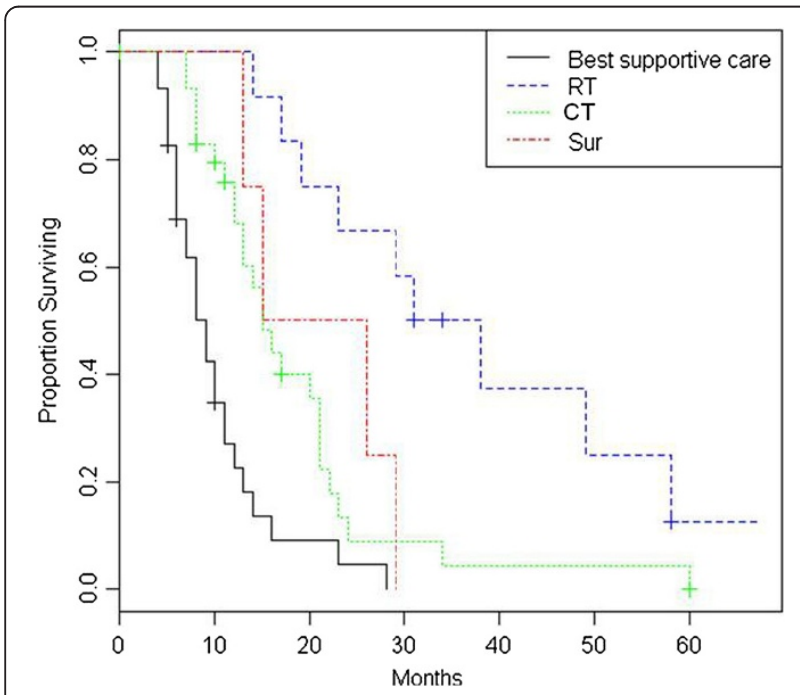

Figure 1 Kaplan-Meier analysis of overall survival from the time of initial diagnosis in patients with recurrent GBM treated with radiotherapy (RT), surgery (Sur), chemotherapy (CT) or best supportive care. exists. Chemotherapy is the most common treatment option for recurrent GBM, but it is associated, when administered alone, with poor OS ( 4-6 months) [41].

The drugs commonly used in the treatment of these patients are many (TMZ, carboplatin, procarbazine, bevacizumab and imatinib) administered as singleagent or in combination. Recent series reported promising results with TMZ alone with a 6 months PFS of $30 \%$ [13]. The use of Bevacizumab was approved by the US Food and Drug Administration because of some phase II trials indicating prolonged 6-month PFS and OS [45]. Several studies suggest that a more aggressive approach with gross total resection and/or high dose re-irradiation may result in improvement of local control of recurrent GBM. A recent review reported an OS of 3-11 months in patients with recurrent GBM treated with surgery [23]. Young et al. [46] reported in 24 patients with tumor recurrence treated with surgery alone an OS of 3.3 months. With a combination of re-intervention and chemotherapy, an increase of PFS and OS was reported [47].

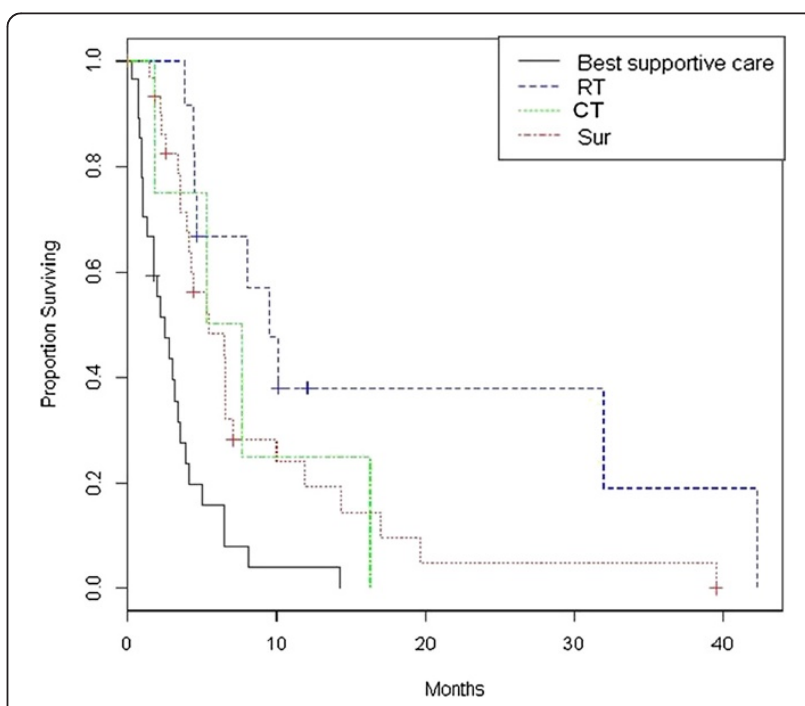

Figure 2 Kaplan-Meier analysis of overall survival from the time of recurrence in patients with recurrent GBM treated with radiotherapy (RT), surgery (Sur), chemotherapy (CT) or best supportive care. 
Table 2 Survey of clinical outcomes after re-irradiation: fractionated stereotactic radiation therapy (FSRT), radiosurgery (SRS), brachytherapy (BT) of recurrent GBM

\begin{tabular}{|c|c|c|c|c|}
\hline Authors & Patients (n) & Type of RT & Total dose/fractions & Outcomes from the re-irradiation \\
\hline Cho et al. [30] & 25 & FSRT & Median dose of 37.5 Gy (range, 20-45 Gy) /2.5 Gy fractions (range, 1.8-3 Gy) & Median survival 12 months \\
\hline Cho et al. [30] & 46 & SRS & Median total dose of 17 Gy delivered to the median of $50 \%$ isodose surface & Median survival 11 months \\
\hline \multirow[t]{4}{*}{ Combs et al. [31] } & 59 & FSRT & 36 Gy/2 Gy fractions & Median OS 8 months \\
\hline & & & & 1 -year survival rates $23 \%$ \\
\hline & & & & Median PFS 5 months \\
\hline & & & & 1-year PFS 5\% \\
\hline Vordermark et al. [33] & 19 & FSRT & Median total dose 30 Gy (range, 20-30 Gy) /5 Gy fractions (range, 4-10 Gy) & Median OS 7.9 months \\
\hline Simon et al. [49] & 42 & Iridium BT & 50 Gy & Median OS 12.5 months \\
\hline Chan et al. [50] & 24 & BT & 53 Gy & Median OS 9.1 months \\
\hline Larson et al. [51] & 14 & SRS & 15 Gy/1 fraction & Median OS 9.5 months \\
\hline Combs et al. [32] & 32 & SRS & 15 Gy/1 fraction & Median OS 10 months \\
\hline Shrieve et al. [52] & 86 & SRS & $13 \mathrm{~Gy} / 1$ fraction & Median OS 10.2 months \\
\hline Shrieve et al. [52] & 32 & BT & 50 Gy & Median OS 11.5 months \\
\hline Grosu et al. [53] & 33 & FSRT & 30 Gy & $\begin{array}{c}\text { Median OS } 8 \text { months (for astrocytomas } \\
\text { and gliomas) }\end{array}$ \\
\hline Kohshi et al. [54] & 25 & FSRT & $22 \mathrm{~Gy}$ & Median OS 11 months \\
\hline \multirow[t]{2}{*}{ Ernst-Stecken et al. [55] } & 15 & FSRT & 35 Gy/7 Gy fractions & 6 months PFS 75\% \\
\hline & & & & 12 months PFS 53\% \\
\hline \multirow[t]{3}{*}{ Fokas et al. [56] } & 53 & FSRT & Median dose 30 Gy (range 20-60 Gy)/ 3 Gy fractions (range 2-5 Gy) & Median OS 9 months \\
\hline & & & & 1-year PFS 22\% \\
\hline & & & & 2-year PFS 5\% \\
\hline Henke et al. [57] & 31 (2 grade III, 29 grade IV) & FSRT & Median total dose 20Gy (range, 20-25)/ 5 Gy fractions & Median OS 10.2 months, \\
\hline Fogh et al. [58] & 147 (42 grade III, 105 grade IV) & FSRT & Median dose 35 Gy in 3.5-Gy fractions & $\begin{array}{c}\text { Median OS } 11 \text { months for grade III and } 8 \\
\text { months for grade IV }\end{array}$ \\
\hline Shepherd et al. [59] & 29 & FSRT & Median dose 35 Gy (range, 20-50 Gy)/ 5 Gy fractions & Median OS 10.7 months \\
\hline Glass et al. [60] & 20 (7 grade III, 13 grade IV) & FSRT & Median dose 38 Gy (range, 35-42 Gy)/ 3.5-6 Gy fractions & Median OS 12.7 months \\
\hline Hudes et al. [61] & 19 & FSRT & Median dose 30 Gy (range, 24-35 Gy)/ 3-3.5 Gy fractions & Median OS 10.5 months \\
\hline Lederman et al. [34] & 88 & FSRT & Total dose 18-36/ 4-9 Gy (weekly) & Median OS 7 months \\
\hline Voynov et al. [62] & 10 (5 WHO grade III, 5 grade IV) & FSRT & 30 Gy /5 Gy fractions & Median OS 10.1 months \\
\hline
\end{tabular}


During the last decade, there has been an increased interest in fractionated stereotactic radiation therapy as a palliative treatment of recurrent GBM.

Combs et al. reported a median OS after re-irradiation of 8 months by applying normo-fractionated stereotactic radiotherapy [32]. The same authors have found an OS of 10 months in patients undergoing single-fraction stereotactic radiosurgery for recurrent GBM [28]. Vordermark et al. [33] showed a median OS of 7.9 months after hypofractionated stereotactic radiation therapy in 19 patients with recurrent GBM. Sirin et al. reported an overall survival from recurrence of 9.3 months after radiosurgery for recurrent GBM [48]. Similar results have been reported by other studies summarized in Table 2 [30-34,49-63]. But a comparison of clinical outcomes provided by the application of so many and different approaches of re-irradiation certainly remains difficult because of the variation in target definition, treatment technique, total dose and dose/ fractions, in the introduction of concomitant chemotherapy and initial patient characteristics.

A recent review has analysed data from more than 300 GBM patients and has demonstrated that re-irradiation yields an increase of the 6-month PFS, that moves from $28 \%$ to $39 \%$ and 1-year overall survival of $18 \%$ to $48 \%$, without additional chemotherapy [64]. A clinical improvement was observed in $24 \%$ to $45 \%$ of the cases with patients with KPS <70 apparently having lesser benefit from re-irradiation. Recently particular attention received the combination of stereotactic radiation therapy and TMZ in recurrent GBM. The use of TMZ in addition to radiotherapy was based on the observation that concurrent chemotherapy can potentiate the cytotoxicity of radiation. In a series of 25 patients with recurrent GBM treated at dose of 36 Gy in 2-Gy fractions in combination with TMZ, the reported median OS and PFS from re-irradiation were 8 and 5 months, respectively [36]. Grosu et al. reported a median survival time of 11 months for patients who have received fractionated stereotactic radiation therapy plus TMZ, while a survival time of 6 months was reached by patients treated with fractionated stereotactic radiation therapy without TMZ $(\mathrm{p}=0.0008)$ [53]. Literature is sparse regarding the toxicity of hypo-fractionated stereotactic radiotherapy in recurrent GBM. Some studies reported higher rates of necrosis but because of the wide range of delivered doses the data are inconclusive. However, the comparison of different studies of re-irradiation remains difficult because of the variability in target definition, treatment technique, fractionation schema, use of concomitant chemotherapy and initial patient characteristics. In the management of $\mathrm{GB}$, the pattern of failure is one of the major concerns in relation to the clinical target volume margins, optimal radiation dose, and identification of biomarkers. In the majority of reported series, local progression after initial management was encountered in $60 \%$ to $97 \%$ of cases, but comparison of different studies is difficult due to differences in treatment strategy, extension of surgical removal, postoperative surveillance, length of follow up as well as definition and categorization of the tumor progression. Several studies have shown that the majority of patients with GBM treated with RT plus concomitant and adjuvant temozolomide have central recurrences [65-68]. Furthermore, many previous trials found that the type of local recurrence in relation to the radiation fields ("in field", "marginal" or "out-field" recurrence) was associated with impaired prognosis [68-73]. In particular, the median survival was $17.3,14.8$ and 26.1 months in patients with recurrence inside, at the margin and outside the irradiation field [71].

The objective of the current study is to evaluate retrospectively the efficacy and the safety of the re-irradiation of patients with recurrent GBM in comparison with other salvage approaches. The patients of the study were part of a consecutive series of 91 patients previously treated with standard RT and TMZ.

In our series the pattern of recurrences evaluated in 71 cases was : "in-field" recurrence in 51 patients (73\%), marginal recurrence in 7 patients $(9 \%)$ and "out-of-field" recurrence in 13 patients (17\%). At tumor recurrence/ progression, only 15 and 6 patients received a salvage radiation treatment or a re-operation, respectively. The remaining 50 patients with disease progression had been considered un-fit for local therapy and received a second line chemotherapy (37 patients) or the best supportive care (13 patients). The median survival time after diagnosis of tumor recurrence in the group of patients treated with re-irradiation (9.5 months) was significantly longer than that who received best supportive care $(2.5$ months, $\mathrm{p}=0.0001$ ) and which has had a longer trend compared to that of patients treated with chemotherapy of second line (5.5 months, $\mathrm{p}=0.049$ ). Furthermore, we have found that patients treated with re-irradiation at the time of tumor recurrence has had the longest overall survival from the time of initial diagnosis (33 months, $\mathrm{p}=0.000049$ ). However, we can not exclude that this finding may result from selection bias for application of the different types of salvage treatment. Also the patients who underwent resurgery show to survive less than re-irradiated patients (median OS 33 vs 17 months respectively, $\mathrm{p}=0.00034$ ). We did not found a significant correlation between the OS and the pattern of failure, although in the group of patients with "in-field" rather than outside progression a mild tendency to a better OS was observed.. This trend might reflect an other selection bias, due to the possible inclusion of some cases of pseudo-progression erroneously interpreted as local recurrence.

With regard to the time interval between the first to the second irradiation, we found a positive but not significant 
correlation between longer interval and survival after reirradiation.

Unfortunately, our study represents a retrospective analysis and patients at the time of tumor recurrence were not randomized to one or the other salvage treatment. In addition, the indication for the salvage treatment and the choice of which perform (surgery, re-irradiation, chemotherapy) was evaluated by our interdisciplinary neurooncology team (and from the patient preferences too). The clinical and tumoral features that have guided their decision were mainly: the patient clinical condition (i.e. KPS> 70), the lesion location and the spread of disease. This means that patients with up-front worse prognosis were excluded from the re-irradiation. Stratifying for these characteristics, the analysis of all patients showed no differences between the groups of different salvage strategies and did not allow the identification of factors associated with choice of one or other salvage treatment. On the basis of these considerations, it was not possible to match the patients included in these groups and therefore the drawing of any conclusions regarding the efficacy of each treatment modality it can suffer of some bias and/or arbitrary.

On the other hand, our results confirm the feasibility and safety of re-irradiation in recurrent GBM. In fact, in our population, although it is a small series, re-irradiation was not accompanied by any case of significant morbidity or side effect. Literature is sparse regarding the toxicity of hypo-fractionated stereotactic radiotherapy in recurrent GBM. Previous studies reported higher rates of necrosis but have utilized a wide range of doses. An association has been noted between higher rates of re-operation and doses greater than 40 Gy [59,74]. With the limitations of the data now at our disposal, radiation therapy for recurrent GBM after standard therapy seems to be safe and in our study the re-irradiated patients have a longer OS compared to patients treated with other approaches.

\section{Conclusion}

Our study shows that hypo-fractionated stereotactic radiation therapy is effective and safe in recurrent GBM after conventional chemo-radiation treatment, even if the dose response and dose limits remain unclear. Anyway, until prospective randomized trials will confirm these results, the decisions for salvage re-irradiation should be based on multidisciplinary evaluation and personalized on the patient.

\section{Competing interests}

All authors disclose no actual or potential conflict of interest including any financial, personal or other relationships with other people or organizations that could inappropriately influence their work.

\section{Authors' contribution}

$P C, A P$ and $C l$ participated in the design of the study. PC, AP, MG, AP and ND carried out the data and participated in the data evaluation. $P C, A B, A P$ and EC performed the statistical analysis. PC and AP drafted the manuscript. The definitive supervision of the paper was done by $\mathrm{Cl}$ and $\mathrm{MI}$. All authors read and approved the final manuscript.

\section{Author details}

${ }^{1}$ Radiation Therapy Unit, Department of Oncology and Advanced Technology, Azienda Ospedaliera ASMN, Istituto di Ricovero e Cura a Carattere Scientifico, Viale Risorgimento 80, 42123 Reggio Emilia, Italy. ${ }^{2}$ Neurology Unit, Dipartimento Neuro-Motorio, Azienda Ospedaliera ASMN, Istituto di Ricovero e Cura a Carattere Scientifico, Viale Risorgimento 80, 42123 Reggio Emilia, Italy. ${ }^{3}$ Medical Physics Unit,Department of Oncology and Advanced Technology, Azienda Ospedaliera ASMN, Istituto di Ricovero e Cura a Carattere Scientifico, Viale Risorgimento 80, 42123 Reggio Emilia, Italy.

Received: 9 July 2013 Accepted: 17 September 2013

Published: 25 September 2013

\section{References}

1. Stupp R, Mason WP, van den Bent MJ, Weller M, Fisher B, Taphoorn MJ, Belanger K, Brandes AA, Marosi C, Bogdahn U, Curschmann J, Janzer RC, Ludwin SK, Gorlia T, Allgeier A, Lacombe D, Cairncross JG, Eisenhauer E, Mirimanoff RO: Radiotherapy plus concomitant and adjuvant temozolomide for glioblastoma. N Engl J Med 2005, 352:987-996.

2. van den Bent MJ, Brandes AA, Rampling $R$, et al: Randomized phase II trial of erlotinib versus temozolomide or carmustine in recurret glioblastoma: EORTC Brain Tumor Group study 26034. J Clin Oncol 2009, 27(8):1268-1274.

3. Brandes AA, Tosoni A, Amista $P$, et al: How effective is BCNU in recurrent glioblastoma in the modern era? A phase II trial. Neurology 2004, 63(7):1281-1284

4. Reithmeier T, Graf E, Piroth T, Trippel M, Pinsker MO, et al: BCNU for recurrent glioblastoma multiforme: efficacy, toxicity and prognostic factord. BMC Cancer 2010 Feb 2, 10:30.

5. Batchelor T, Mulholland P, Neyns B, et al: The efficacy of cediranib as monotherapy and in combination with lomustine compared to lomustine alone in patients with recurrent glioblastoma: a phase III randomized trial. Neuro Oncol 2010, 12(Suppl. 4):75.

6. Happold C, Roth P, Wick W, et al: ACNU-based chemotherapy for recurrent glioma in the temozolomide era. J Neurooncol 2009, 92(1):45-48.

7. Addeo R, Caraglia M, De Santi MS, et al: A new schedule of fotemustine in temozolomide-pretreated patients with relapsing glioblastoma. J Neuroncol 2011, 102(3):417-424.

8. Scoccianti S, Detti B, Sardaro A, et al: Second-line chemotherapy with fotemustine in temozolomide-pretreated patients with relapsing glioblastoma: a single institution experience. Anticancer Drugs 2008, 19(6):613-620.

9. Brada M, Hoang-Xuan K, Rampling R, et al: Randomized phase II trial of temozolomide in patients with glioblastoma multiforme at first relapse. Ann Oncol 2001, 1282:259-266.

10. Brandes AA, Ermani M, Basso U, et al: Temozolomide as a second-line sistemi regimen in recurrent high-grade glioma: a phase II study. Ann Oncol 2011, 12(2):255-257.

11. Brandes AA, Ermani M, Basso U, et al: Temozolomide in patients with glioblastoma at second relapse after first line nitrosurea-procarbazine failure: a phase II study. Oncology 2002, 63(1):38-41.

12. Khan RB, Raizer JJ, Malkin MG, et al: A phase II study of extended low-dose temozolomide in recurrent malignant gliomas. Neuro Oncol 2002, 4(1):39-43.

13. Brandes AA, Tosoni A, Cavallo G, et al: Temozolomide 3 weeks on and 1 week off as first-line therapy for recurrent glioblastoma: phase II study from Gruppo Italiano Cooperativo di Neuro-oncologia (GICNO). Br J Cancer 2006, 95(9):1155-1160.

14. Balmaceda C, Peereboom D, Pannullo S, et al: Multi-institutional phase II study of temozolomide administered twice daily in the treatment of recurrent high-grade gliomas. Cancer 2008, 112(5):1139-1146.

15. Wick W, Steinbach JP, Kuker WM, et al: One week on/one week off: a novel active regimen of temozolomide for recurrent glioblastoma. Neurology 2004, 62(11):2113-2115

16. Yung WK, Albright RE, Olson J, et al: A phase II study of temozolomide vs. procarbazine in patients with glioblastoma multiforme at first relapse. Br J Cancer 2000, 83(5):588-593.

17. Franceschi E, Omuro AM, Lassman AB, Demopoulos A, et al: Salvage temozolomide for prior temozolomide responders. Cancer 2005, 104(11):2473-2476. 
18. Kong DS, Lee Jl, Kim WS, et al: A pilot study of metronomic temozolomide treatment in patients with recurrent temozolomide-refractory glioblastoma. Oncol Rep 2006, 16(5):117-1121.

19. Berrocal A, Perez Segura P, Gil M, et al: Extended-schedule dose-dense temozolomide in refractory gliomas. J Neuroncol 2010, 96(3):417-422.

20. Kong DS, Lee J, Kim JH, et al: Phase II trial of low-dose continuous (metronomic) treatment of temozolomide for recurrent glioblastoma. Neuro Oncol 2010, 12(3):289-296.

21. Hammond A, Norden AD, Lesser GJ, et al: Phase II study of dose-intense temozolomide in recurrent gliblastoma. J Clin Oncol 2038, 2011:29.

22. Park JK, Hodges T, Arko L, et al: Scale to predict survival after surgery for recurrent glioblastoma multiforme. J Clin Oncol 2010, 28(24):3838-3843.

23. Barbagallo GM, Jenkinson MD, Brodbelt AR: Recurrent Gliobastoma multiforme- when should we reoperate? Br J Neurosurg 2008, 22(3):452-455

24. Dhermain F, de Crevoisier R, Parker F, Cioloca C, Kaliski A, Beaudre A, Lefkopoulos D, Armand JP, Haie-Meder C: Role of radiotherapy in recurrent gliomas. Bull Cancer 2004, 91(11):883-889.

25. Coombs SE, Debus J, Shulz-Ertner D: Radiotherapeutic alternatives for previously irradiated recurrent gliomas. BMC Cancer 2007, 7:167.

26. Hall WA, Djalilian HR, Sperduto PW, et al: Stereotactic radiosurgery for recurrent malignant gliomas. J Clin Oncol 1995, 13:1642-1648.

27. Cabrera AR, Cuneo KC, Vredenburgh JJ, et al: Stereotactic radiosurgery and bevacizumab for recurrent glioblastoma multiforme. J Natl Compr Canc Netw 2012 Jun 1, 10(6):695-699.

28. Combs SE, Widmer V, Thilmann C, et al: Stereotactic radiosurgery (SRS): treatment option for recurrent glioblastoma multiforme (GBM). Cancer 2005, 104:2168-2173

29. Patel $M$, Siddiqui $F$, Jin JY, Mikkelsen $T$, et al: Salvage reirradiation for recurrent glioblastoma with radiosurgery: radiographic response and improved survival. J Neurooncol 2009, 92:185-191.

30. Cho KH, Hall WA, Gerbi BJ, Higgins PD, et al: Single dose versus fractionated stereotactic radiotherapy for recurrent high-grade gliomas. Int J Radiat Oncol Biol Phys 1999, 45:1133-1141.

31. Combs SE, Gutwein S, Thilman CH, et al: Stereotactically guided fractionated reirradiation in recurrent glioblastoma multiforme. J Neurooncol 2005, 74:167-171.

32. Combs SE, Thilman $\mathrm{CH}$, Edler $\mathrm{L}$, et al: Efficacy of fractionated stereotactic reiraddiation in recurrent gliomas: long-term result in 172 patients treated in a single institution. J Clin Oncol 2005, 23:8863-8869.

33. Vordermark D, Kolbl O, Ruprecht $\mathrm{K}$, et al: Hypofractionated stereotactic re-irradiarion: treatment option in recurrent malignant glioma. BMC Cancer 2005, 5:55

34. Lederman $\mathrm{G}$, Wronski M, Arbit $\mathrm{E}$, et al: Treatment of recurrent glioblastoma multiforme using fractionated stereotactic radiosurgery and concurrent paclitaxel. Am J Clin Oncol 2000, 23:155-159.

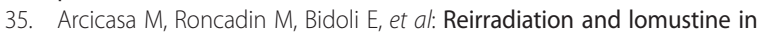
patients with relapsed high-grade gliomas. Int J Radiat Oncol Biol Phys 1999, 43:789-793.

36. Combs SE, Bischof M, Welzel T, et al: Radiochemotherapy with temozolomide as re-irradiation using high precision fractionated stereotactic radiotherapy (FSRT) in patients with recurrent gliomas. J Neurooncol 2008, 89:205-210.

37. Minniti $G$, Armosini $V$, Salvati $M$, et al: Fractionated stereotactic reirradiation and concurrent temozolomide in patients with recurrent glioblastoma. J Neurooncol 2011, 103(3):683-691.

38. Louis DN, Ohgaki H, Wiestler OD, Cavenee WK: WHO Classification of Tumours of the Central Nervous System. IARC: Lyon; 2007.

39. Lee SW, Fraas BA, Marsh LH, et al: Patterns of failure following hig-dose 3-D conformal radiotherapy for high-grade astrocytomas: A quantitative dosimetric study. Int J Radiat Oncol Biol Phys 1999, 43(199):79-88.

40. Ammirati M, Galicich JH, Arbit E, Liao Y: Reoperation in the treatment of recurrent intracranial malignant gliomas. Neurosurgery 1987, 21:607-614.

41. Hou LC, Veeravagu A, Hsu AR, Tse VC: Recurrent glioblastoma multiforme: a review of natural history and management options. Neurosurgical focus 2006, 20:E5.

42. Nieder C, Grosu AL, Molls M: A comparison of treatment results for recurrent malignant gliomas. Cancer Treat Rev 2000, 26:397-409.

43. Stupp R, Hegi ME, Gilbert MR, et al: Chemoradiotherapy in malignant glioma: standard of care and future directions. J Clin Oncol 2007, 25:4127-4136.

44. Brandes AA, Franceschi E, Tosoni A, Bartolini S, Bacci A, Agati R, et al: O(6)-methylguanine DNA-methyltransferase methyleation status can change between first surgery for newly diagnosed glioblastoma and second surgery from recurrence: clinical implications. Neuro Oncol 2010, 12:283-288.

45. Vredenburgh JJ, Desjardinis A, Herndon JE 2nd, et al: Bevacizumab plus irinotecan in recurrent glioblastoma multiforme. J Clin Oncol 2007 25:4722-4729

46. Young B, Oldfield EH, Markesbery WR, Haack D, Tibbs P, McCoombs P, et al: Reoperation for glioblastoma. J Neurosurg 1981, 55:917-921.

47. Barker FG 2nd, Chang SM, Gutin PH, Malec MK, McDermott MW, Prados MD, et al: Survival and functional status after resection of recurrent glioblastoma multiforme. Neurosurgery 1998, 42:709-720.

48. Sirin S, Oysul K, Surenkok S, et al: Linear accelerator-based stereotactic radiosurgery in recurrent glioblastoma: a single center experience. Vojnosanit Pregl 2011, 68(11):961-6.

49. Simon JM, Cornu P, Boisserie G, et al: Brachytherapy of glioblastoma recurring in previously irradiated territory: predictive value of tumor volume. Int J Radiat Oncol Biol Phys 2002, 53:67-74.

50. Chan TA, Weingart JD, Parisi M, et al: Treatment of recurrent glioblastoma multiforme with GliaSite brachytherapy. Int J Radiat Oncol Biol Phys 2005 62:1133-1139.

51. Larson DA, Prados M, Lamborn KR, et al: Phase II study of high central dose gamma knife radiosurgery and marimastat in patients with recurrent malignant glioma. Int J Radiat Oncol Biol Phys 2002, 54:1397-1404.

52. Shrieve DC, Alexander E 3rd, Wen PY, et al: Comparison of stereotactic radiosurgery and brachytherapy in the treatment of recurrent glioblastoma multiforme. Neurosurgery 1995, 36:275-282. discussion 282-4

53. Grosu AL, Weber WA, Franz $M$, et al: Reirradiation of recurrent high-grade gliomas using amino acid PET (SPECT)/CT/MRI image fusion to determine gross tumor volume for stereotactic fractionated radiotherapy. Int J Radiat Oncol Biol Phys 2005, 63:511-519.

54. Kohshi K, Yamamoto H, Nakahara A, et al: Fractionated stereotactic radiotherapy using gamma unit after hyperbaric oxygenation on recurrent high-grade gliomas. J Neurooncol 2007, 82:297-303.

55. Ernst-Stecken A, Ganslandt O, Lambrecht U, Sauer R, Grabenbauer G: Survival and quality of life after hypofractionated stereotactic radiotherapy for recurrent malignant glioma. J Neurooncol 2007, 81:287-294.

56. Fokas E, Wacker U, Gross MW, Henzel M, Encheva E, Engenhart-Cabillic R: Hypofractionated stereotactic reirradiation of recurrent glioblastomas: a beneficial treatment option after high-dose radiotherapy? Strahlenther Onkol 2009, 185:235-240.

57. Henke G, Paulsen F, Steinbach JP, Ganswindt U, Isijanov H, Kortmann RD, Bamberg M, Belka C: Hypofractionated reirradiation for recurrent malignant glioma. Strahlenther Onkol 2009, 185:113-119.

58. Fogh SE, Anrews DW, Glass J, Curran W, Glass C, Champ C, Evans JJ, Hyslop T, Pequignot E, Downes B, Comber E, Maltenfort M, Dicker AP, Werner-Wasik $M$ : Hypofractionated stereotactic radiation therapy: an effective therapy for recurrent high-grade gliomas. J Clin Oncol 2010, 28:3048-3053.

59. Shepherd SF, Laing RW, Cosgrove VP, Warrington AP, Hines F, Ashley SE, Brada M: Hypofractionated stereotactic radiotherapy in the management of recurrent glioma. Int J Radiat Oncol Biol Phys 1997, 37:393-398.

60. Glass J, Silverman CL, Axelrod R, Corn BW, Andrews DW: Fractionated stereotactic radiotherapy with cis-platinum radiosensitization in the treatment of recurrent, progressive or persistent malignant astrocytoma. Am J Clin Oncol 1997, 20:226-229.

61. Hudes RS, Corn BW, Werner-Wasik M, Andrews D, Rosenstock J, Thoron L, Downes B, Curran WJ: A phase I dose escalation study of hypofractionated stereotactic radiotherapy as salvage therapy for persistent or recurrent malignant glioma. Int J Radiat Oncol Biol Phys 1999, 43:291-298

62. Voynov G, Kaufman S, Hong T, Pinkerton A, Simon R, Dowsett R: Treatment of recurrent malignant gliomas with stereotactic intensity modulated radiation therapy. Am J Clin Oncol 2002, 25:606-611.

63. Veninga $T$, Langendijk HA, Slotman BJ, et al: Reirradiation of primary brain tumors: serviva, clinical response and prognostic factors. Rdaiother Oncol 2001, 59:127-137.

64. Nieder C, Astner ST, Mehta MP, et al: Improvement, clinical course, and quality of life after palliative radiotherapy for recurrent glioblastoma. Am J Clin Oncol 2008, 31:300-305.

65. Chang EL, Akurek S, Avalos T, et al: Evaluation of peritumoral edema in the delineation of radiotherapy clinical target volumes for glioblastoma. Int J Radiat Oncol Biol Phys 2007, 86:144-150. 
66. Liang BC, Thornton AF Jr, Sandler HM, et al: Malignant atrocytomas: focal tumor recurrence after local external beam radiation therapy. J Neurosurg 1991, 75:559-563.

67. Lee SW, Fraass BA, Marsh LH, et al: Patterns of failure following high-dose 3-D conformal radiotherapy for high-grade astrocytomas: A quantitative dosimetric study. Int J Radiat Oncol Biol Phys 1999, 43:79-88.

68. McDonald MW, Shu HK, Curran WJ, et al: Pattern of failure after limited margin radiotherapy and temozolomide for glioblastoma. Int I Radiat Oncol Biol Phys 2011, 79:130-136.

69. Minniti $G$, Amelio D, Amichetti M, et al: Patterns of failure and comparison of different target volume delineations in patients with gliobalstoma treated with conformal radiotherapy plus concomitant and adjuvant temozolomide. Rdaiother Oncol 2010, 97:377-381.

70. Milano MT, Okunieff P, Donatello RS, et al: Patterns and timing of recurrence after temozolomide-based chemoradiation for glioblastoma. Int J Radiat Oncol Biol Phys 2010, 78:1147-1155.

71. Brandes AA, Tosoni A, Franceschi E, et al: Recurrence pattern after temozolomide concomitant with and ajuvant to radiotherapy in newly diagnosed patients with glioblastoma: correlation with MGMT promoter methylation status. J Clin Oncol 2009, 27:1275-1279.

72. Hochberg FH, Pruitt A: Assumption in the radiotherapy glioblastoma. Neurology 1980, 30:907-911.

73. Stewart LA: Chemotherapy in adult high-grade glioma: A systematic review and meta-analysis of individual patient data from 12 randomised trials. Lancet 2002, 359:1011-1018.

74. Laing RW, Warrington AP, Graham J, et al: Efficacy and toxicity of fractionated stereotactic radiotherapy in the treatment of recurrent gliomas (Phase I//l study). Radiother Oncol 1993, 27:22-29.

doi:10.1186/1748-717X-8-222

Cite this article as: Ciammella et al:: Hypofractionated stereotactic radiation therapy for recurrent glioblastoma: single institutional experience. Radiation Oncology 2013 8:222.

\section{Submit your next manuscript to BioMed Central and take full advantage of:}

- Convenient online submission

- Thorough peer review

- No space constraints or color figure charges

- Immediate publication on acceptance

- Inclusion in PubMed, CAS, Scopus and Google Scholar

- Research which is freely available for redistribution 